\title{
Auto-focus system based on general hardness tester
}

\author{
Kunyang Cao ${ }^{1, \text { a) }}$, Xiaodong $\mathrm{Lv}^{1)}$, Jingtao Wang ${ }^{2)}$, Yimin Dong ${ }^{2)}$, Xiuchun $\mathrm{Li}^{2)}$ \\ ${ }^{1}$ School of Mechanics and Automation, Beihang University, Beijing 100191, China. \\ ${ }^{2}$ Laizhou Huayin Testing Instrument Co., Ltd. Laizhou 261400, Shandong,China. \\ a)michael_cky@buaa.edu.cn
}

Abstract: Manual focus is general adapted in the traditional hardness tester, while the manual focus depends much on the tester, and the precision of the manual varies from tester to tester. Based on the basic principle of image processing of automatic focus, this paper presents auto-focus system applying a chip of STM32F407 as the host controller of hardness tester. Through the test, the system can get the focus position efficiently, and the accuracy is guaranteed.

Keywords: focus, hardness tester, function

\section{INTRODUCTION}

With the rapid development of automation and Intelligence of imaging device, auto-focus is used more and more in many areas, the general hardness tester also develops towards the automatic and intelligent direction. There are two methods of focusing, including manual focus and autofocus [1-2]. Testers adjust the focal length mainly based on their subjective feelings, this method can achieve higher accuracy under the operation of the professional, while that is not guaranteed under the operation of the non-professional. Therefore, the precision of manual focus has significant dependence on the tester, and the effect of the manual varies from tester to tester. The basic principle of autofocus based on the image processing is to process the digital image information collected, to calculate the clarity evaluation value of the image through some algorithm, to determine the imaging clarity and out-of-focus status of the system. According to the evaluation value, the host controller gives feedback signal to drive the focus mechanism to control the movement of the objective table until achieving the image. From this principle, the image method can focus on the full use of the rapidity and flexibility of computer technology to deal with digital signals, and do not need the target location, optical system focal length and other information. With reducing intermediate links and the advantages of fast and accurate [3], the accuracy of the focus is not different from the tester.

\section{THE CONSTRUCT AUTOMATIC FOCUSING SYSTEM}

The system mainly consists of stepper motor drive module, LCD (liquid crystal display) touch screen display module and image acquisition module. The STM32F407ZGT6 is used as the master processor of hardness tester, and this chip has sufficient interfaces and peripheral functions. The stepper motor is a solenoid actuator of converting a certain sequence of electrical pulse signals into angular displacements or linear displacement. As a key part of the field of automation, motion control technology based on stepping motor is widely used in digital control and computer control and other control system of precise positioning [4]. The connection between MCU (Microprogrammed Control Unit) and stepper motor drive uses the general common cathode wiring. The pulse signal required to drive the stepper motor is generated by the MCU internal setting, the driving circuit of the stepping motor operates according to the control signal, controlling the movement direction of the motor by positive sequence or reverse order commutation. LCD touch screen driven by the master chip, is mainly responsible for realtime display images and interface operations.

The image acquisition module mainly acquires the image data by reading the OV2640 image sensor and sends it to the controller for processing to judge whether the focus is successful and gives the feedback signal to drive the movement of the stage. The image sensor controlled by the SCCB bus (Serial Camera Control Bus) can output a variety of resolutions of 8/10 bits' image data of the entire frame, sub-sampling, scaling and windowing, etc. and the output rate of UXGA (Ultra Extended Graphics Array) image can be up to 15 frames /sec (SVGA (Super Video Graphics Array) up to 30 frames, CIF (Graphics Interchange Format) up to 60 frames). The module is connected to the master chip via a $2 * 9$ double row pin. The master chip used by the system comes with DCMI (digital camera interface), which is a synchronous parallel interface that can receive high-speed data streams of 8-bit, 10-bit, 12-bit, or 14-bit CMOS camera modules from external. DCMI Schematic diagram is shown in Fig. 1. 


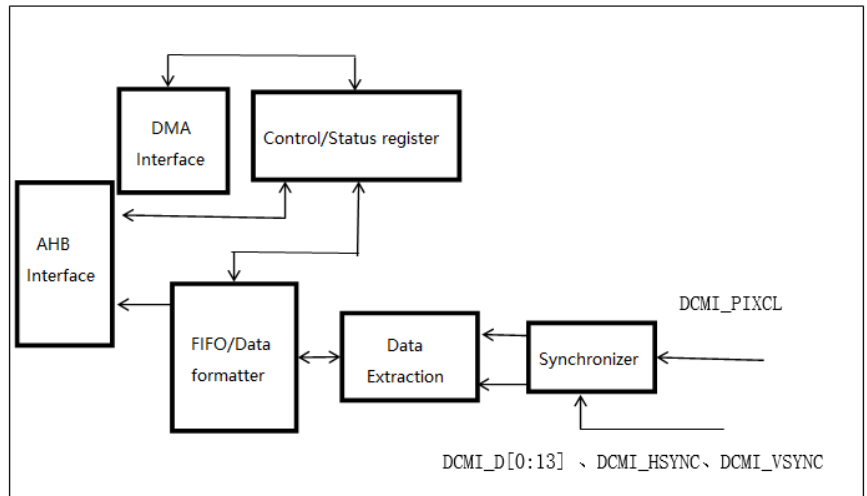

FIGURE 1. DCMI Schematic diagram

\section{THE ALGORITHM OF AUTOMATIC FOCUSING}

At present, the focusing function proposed at home and abroad can be summed up as grayscale gradient function, frequency domain function, informatics function and statistical function [5-7], among which frequency domain class, informatics and statistical focus function require higher the stability of the environment during the focusing process, and in consequence the focus curve is far from ideal, most of them cannot express the location of the focus accurately. The system mainly uses the following gray scale gradient evaluation function:

(1) Mean square function:

$$
\begin{aligned}
& \mathrm{F}=\sum_{x} \sum_{y}\left\{\left[(g(x+1, y)-u)^{2}-(g(x, y)-u)^{2}\right] / 2\right\}^{\frac{1}{2}} \\
& \mathrm{u}=[\mathrm{g}(\mathrm{x}, \mathrm{y})+\mathrm{g}(\mathrm{x}+1, \mathrm{y})] / 2
\end{aligned}
$$

(2) Absolute variance function:

$$
\mathrm{F}=\sum_{x} \sum_{y}|g(x, y)-g(x+1, y)|
$$

(3) Roberts gradient and function:

$$
\mathrm{F}=\sum_{x} \sum_{y}\{|g(x, y)-g(x+1, y+1)|+|g(x+1, y)-g(x, y+1)|\}
$$

(4) Gradient vector modular function:

$$
\mathrm{F}=\sum_{x} \sum_{y}\left\{[g(x+1, y)-g(x, y)]^{2}-[g(x, y+1)-g(x, y)]^{2}\right\}^{\frac{1}{2}}
$$

$$
\mathrm{F}=\sum_{x} \sum_{y}\left\{[g(x+1, y)-g(x, y)]^{2}-[g(x, y+1)-g(x, y)]^{2}\right\}
$$

$$
\mathrm{F}(\mathrm{k})=\sum_{x} \sum_{y}[g(x+k, y)-g(x, y)]^{2}
$$

The system uses the above evaluation function to test, taking into account the speed and efficiency of data processing of the selected chip, the image focus range is selected as shown in Fig. 2, where the image size is outputted by the sensor according to the need to set when initialization. Part A presents the size of the display window, that is, the LCD touch screen window, the image of the sensor zooms in accordance with the window $\mathrm{A}$ ratio. Part B presents the size of the focus window. The image center area is generally the interested and effective region, selecting the whole frame image as the focus window, on the one hand will aggravate the efficiency and speed of the processor; the other hand, taking into account of the experimental operating environment, the difference of brightness between the image center area and edge affects the accuracy of the focus and in consequence the image center is selected as the focus window. 


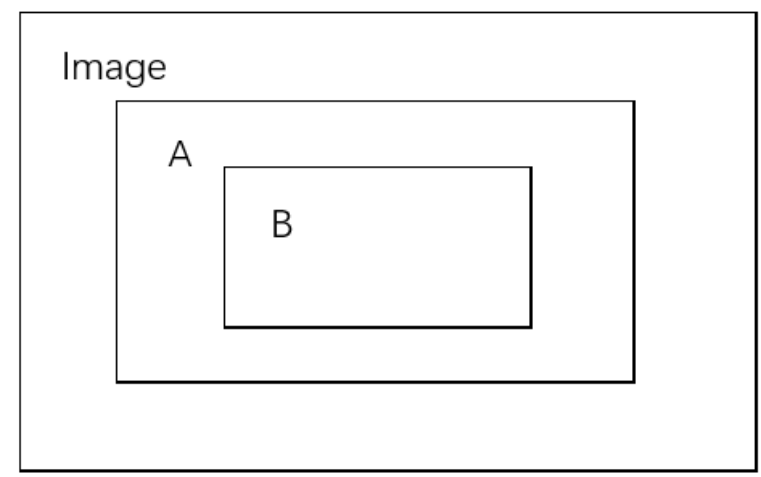

FIGURE 2. Focus window

\section{THE EXPERIMENTAL ANALYSIS}

The ideal evaluation function should be unbiased, unimodal, anti-noise, according to the formula 1-6, data of evaluation functions are outputted through the serial port in the test process. The evaluation function curve is shown in Fig. 3, and each the evaluation function curve basically satisfies the basic characteristics of the above evaluation function. The clearer the image is, the greater the value of the resolution evaluation function is; therefore, the maximum value of the focus curve corresponds to the focus position. $\varepsilon$ is the deviation between the actual focus position P0 and the focal position P2 positioned according to the evaluation function, and the smaller the $\varepsilon$ value is, the better the evaluation function is.

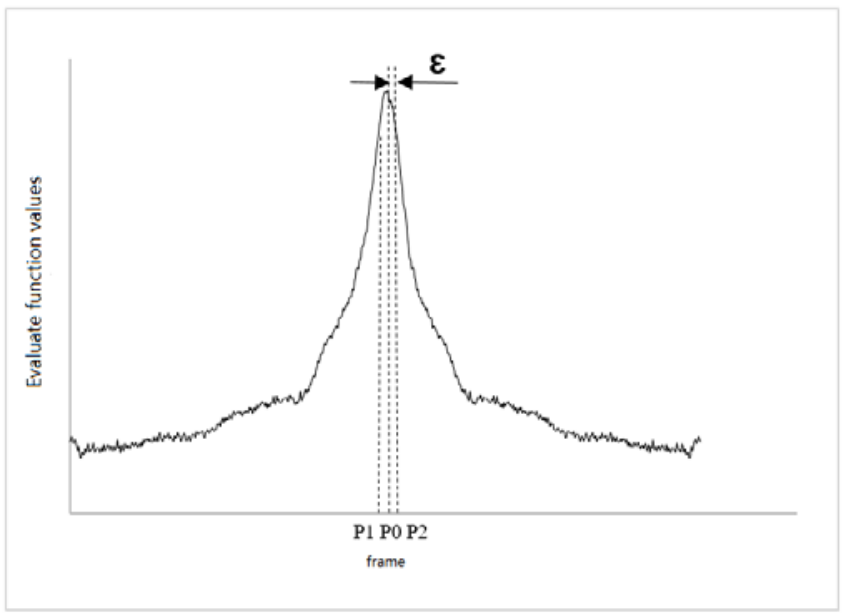

FIGURE 3. Evaluation function curve

Fig. 4 shows the evaluation function curve of focusing. The ABCD segment is a graph of the normal evaluation function. The host controller sends feedback signal to drive the movement of the stage through processing data collected by the image sensor. In the actual focusing process, the evaluation function will be subject to noise and other external environment interference, the initial section of the gentle zone fluctuations, which will interfere with the focusing process and make it fail to search the best focus position. The exhaustive search method is used to avoid the miss of real focus image in the search process. At the beginning of the focus, the objective table moves, and the image value is recorded at any time. The objective table keeps moving when image value reaches to its peak, and stops at $60 \%$ of the maximum, that is, position D in Fig. 4. The threshold of 0.6 is to be able to search for a sufficiently long range of images to eliminate the influence of the noise in the initial section on the focusing process. $\mathrm{F}(\mathrm{C})$ presents the evaluation function value of the theoretical focus, and $\mathrm{F}(\mathrm{E})$ presents the evaluation function value of the actual focus. To maximize the accuracy, test should satisfy the condition: $\mathrm{F}(\mathrm{E}) \geq \mathrm{F}(\mathrm{C}) * 99 \%$. 


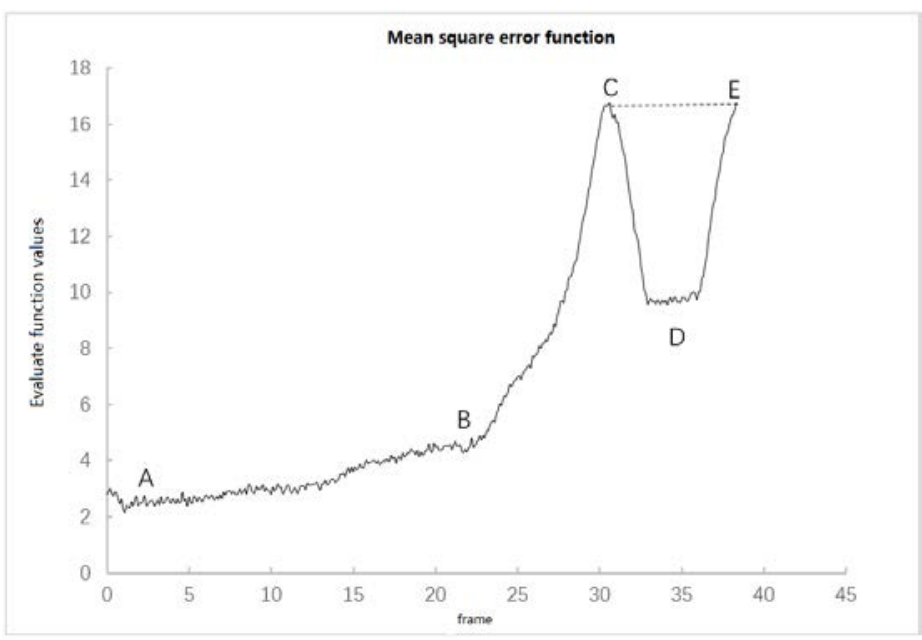

FIGURE 4. Focus curve

According to the above experimental method, the focusing curves of the listed evaluation function are shown in Fig. 5. As can be seen from the focusing curve of the evaluation function, the focusing effect of the Roberts gradient function requiring several times to focus accurately is not satisfied, while the absolute variance function and the gradient vector modular function fail to focus. The mean square error function, the gradient vector squared function and the Brenner function succeed in focusing and have a better effect. 

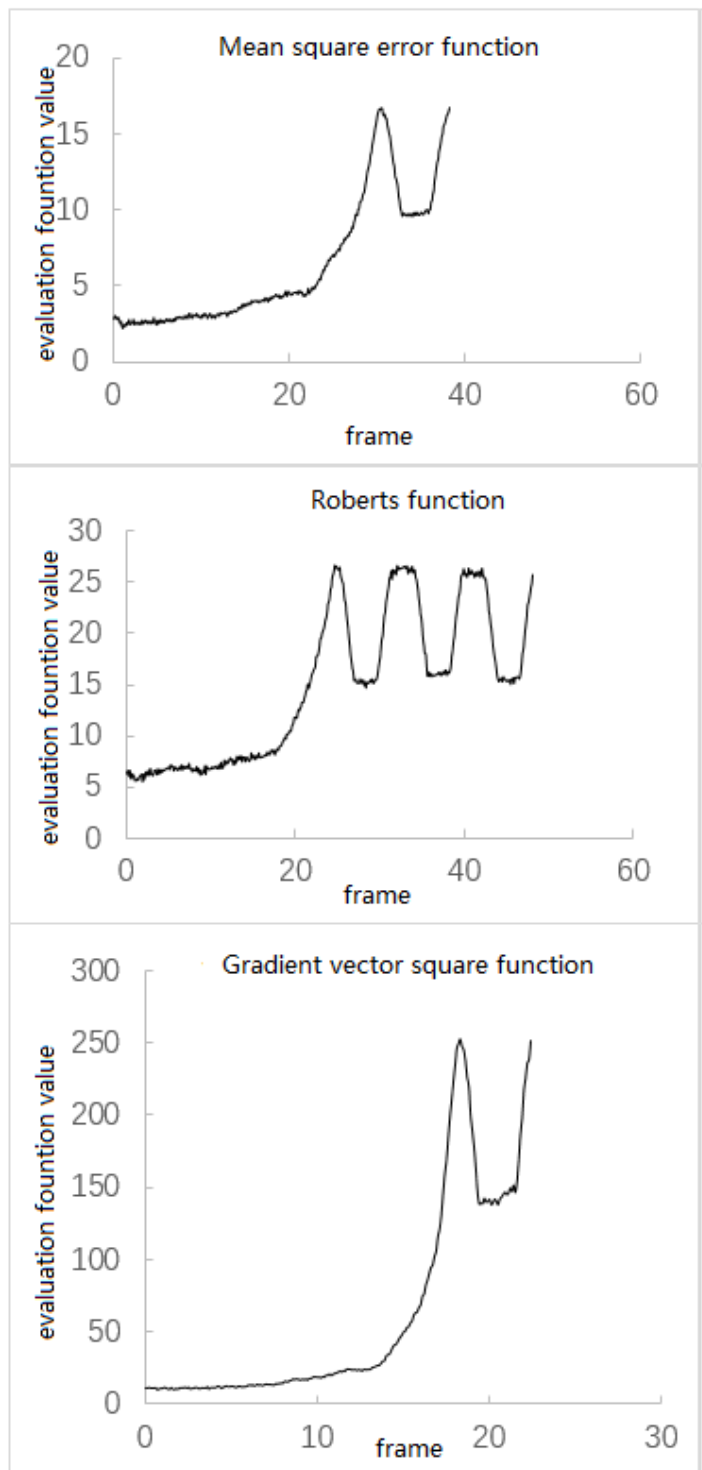
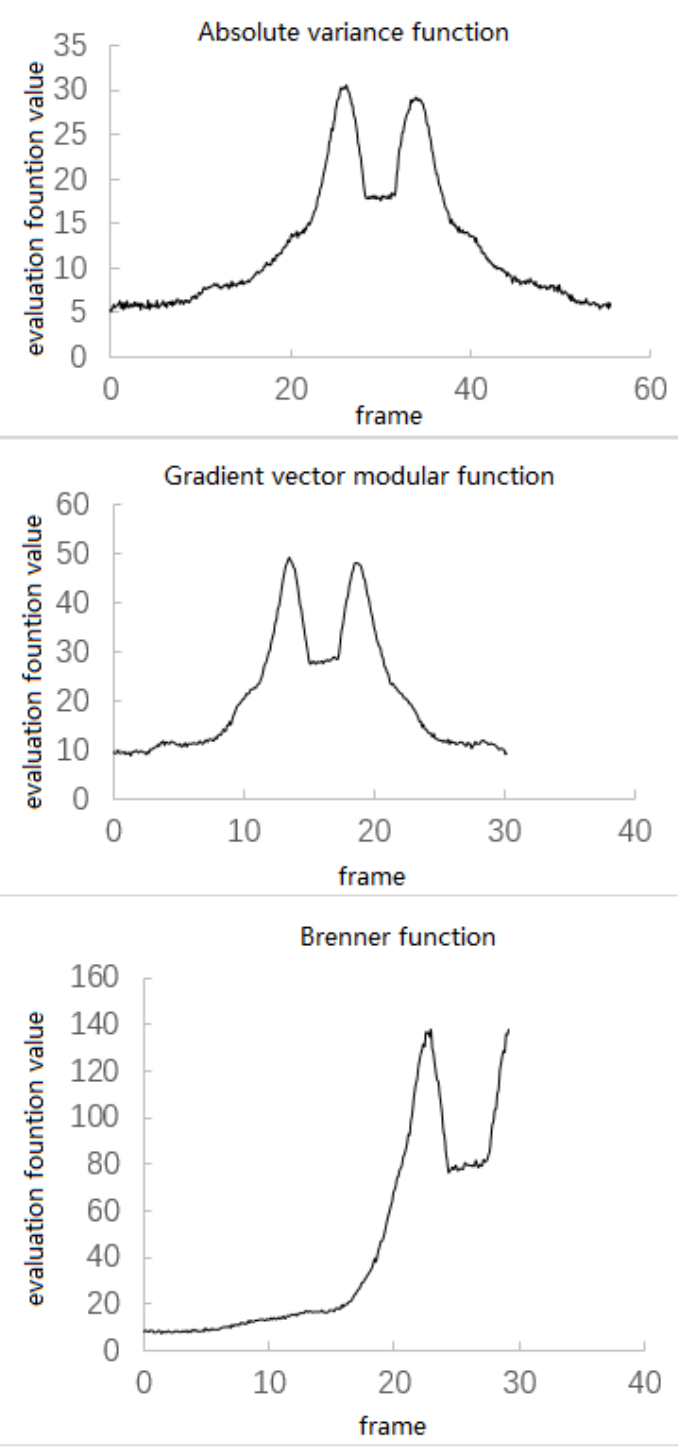

FIGURE 5. Different evaluation function focus curve

It can be seen from Fig. 5 that these three evaluation functions satisfy several significant characteristics of the focusing evaluation function. However, each of the curves identified in the figure is in a different range of values. Characteristic difference in the vicinity of the focus position cannot be reflected only in this way. By the use of normalization, the value of focus functions is limited to between 0 to 1 . Half of the focusing curve is shown in Fig. 6 for the ease of analysis. It is clear to see the different rate of change of the focus curves; the steeper curves indicate the higher sensitivity. It can be obtained from the Fig. 6 that the mean square function has the best focusing performance, followed by the Brenner function, and the focusing function of the gradient vector square function is slightly worse. 


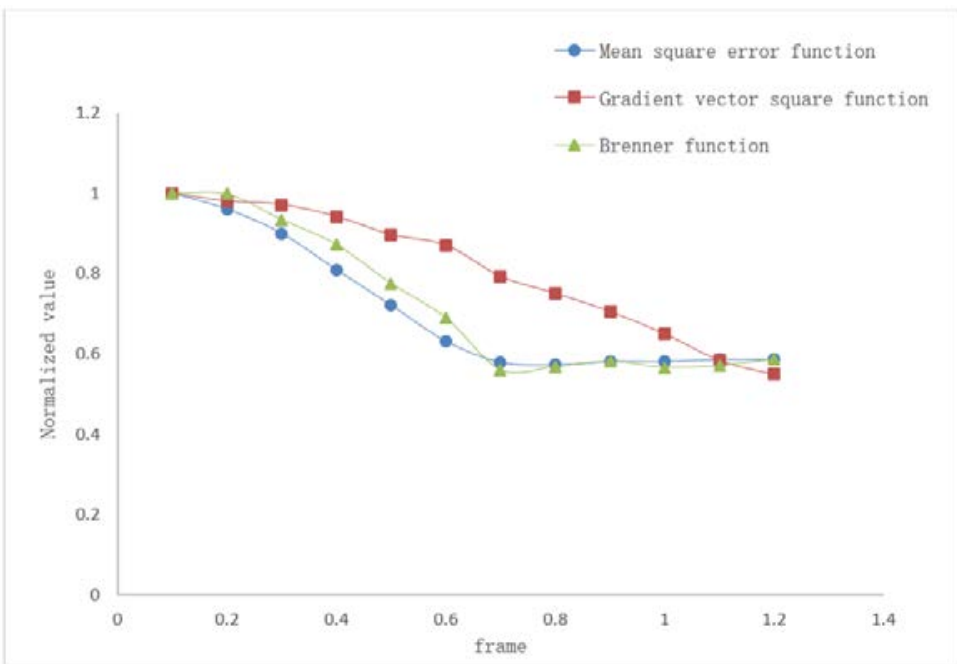

FIGURE 6. Focus curve

The focal length is fixed for the same focus test. Selecting the same focus start position, the graph of the comparison with the calculation efficiency of the above three evaluation functions is shown in Fig. 7, and the abscissa indicates the number of captured images. In the same time, the more frames are acquired, the higher the computational efficiency is and the better the focusing effect is. It can be seen from the graph, the mean square error function acquires 155 images to the focus position, signifying the highest efficiency, and the following is the Brenner function capturing 145 images, while the gradient vector square function only obtains 92 images, and the focus effect is slightly worse.
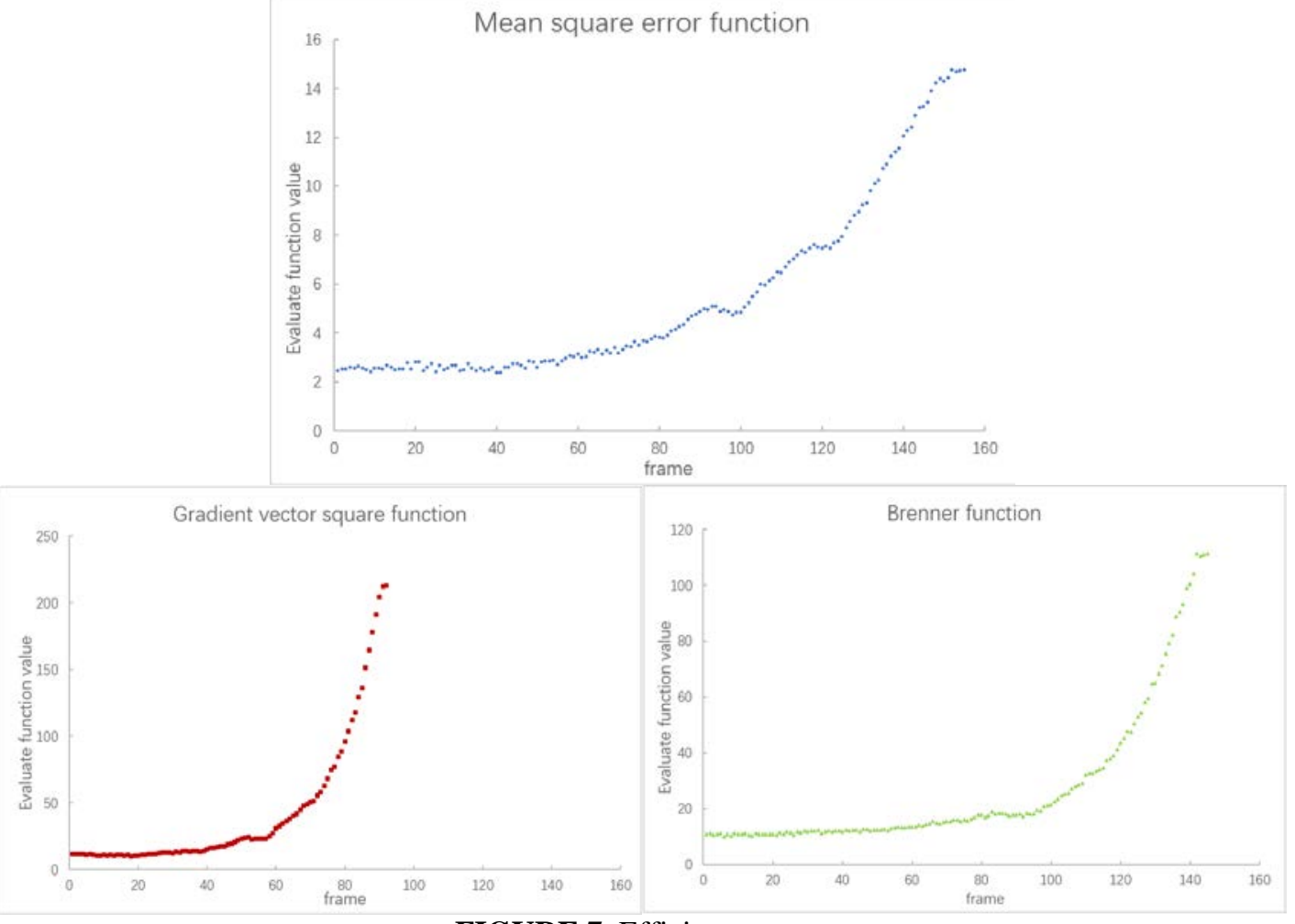

FIGURE 7. Efficiency curve 


\section{CONCLUSION}

This paper presents an experimental platform of automatic focusing system using STM32F407ZGT6 as the core processor, and validates the proposed algorithm. The experimental results show that the absolute variance function, the Roberts gradient function and gradient vector modular function cannot focus accurately. From the comparison of the sensitivity and computational efficiency of the three evaluation functions, the mean square error function, the gradient vector squared function and the squared function focus precisely, and the mean square function has the best focusing performance, followed by the Brenner function, and the focusing function of the gradient vector square function is slightly worse.

\section{REFERENCES}

1. Loren Shih. Autofocus survey: a comparison of algorithms[C]. Proc. SPIE,2007,6502:1-11.

2. H WANG. Principle and method of automatic focusing [J]. Science \& Technology Information,2008, (13):38. (in Chinese)

3. T D HUANG. Study on Auto-focus Method Using Image Technology[D]. Changchun Institute of Optics, Fine Mechanics and Physics Chinese Academy of Sciences, 2013. (in Chinese)

4. Z P WANG. The development review of stepper motor[J]. Micromotors, 2003,3. (in Chinese)

5. J M WANG. Research of 2-D image measuring machine and on-line measuring system for large size arclength[D]. Thesis of Harbin Institute of Technology, 1998. (in Chinese)

6. S G QIU, J ZHOU, X LIU. Research of sharpness evaluation function in array pixel device projection display's auto-focusing system[J]. Acta Optica Sinica, 2004, 24(4):460-464. (in Chinese)

7. J XU, ZH J ZHANG, L H LU. Image processing for the automatic positioning system of pellet target[J]. Optics and Precision Engineering, 2001, 9(6): 568-57. (in Chinese) 